\title{
Photoacoustic imaging of vascular networks in transgenic mice
}

\author{
J.G. Laufer ${ }^{1}$, J.O. Cleary ${ }^{1,2}$, E.Z. Zhang ${ }^{1}$, M.F. Lythgoe ${ }^{2}$, P.C. Beard ${ }^{1}$ \\ 1. Department of Medical Physics and Bioengineering, University College London \\ 2. Centre for Advanced Biomedical Imaging, \\ Department of Medicine and UCL Institute of Child Health, University College London \\ http://www.medphys.ucl.ac.uk/research/mle/index.htm
}

\begin{abstract}
The preferential absorption of near infrared light by blood makes photoacoustic imaging well suited to visualising vascular structures in soft tissue. In addition, the spectroscopic specificity of tissue chromophores can be exploited by acquiring images at multiple excitation wavelengths. This allows the quantification of endogenous chromophores, such as oxy- and deoxyhaemoglobin, and hence blood oxygenation, and the detection of exogenous chromophores, such as functionalised contrast agents. More importantly, this approach has the potential to visualise the spatial distribution of low concentrations of functionalised contrast agents against the strong background absorption of the endogenous chromophores. This has a large number of applications in the life sciences. One example is the structural and functional phenotyping of transgenic mice for the study of the genetic origins of vascular malformations, such as heart defects. In this study, photoacoustic images of mouse embryos have been acquired to study the development of the vasculature following specific genetic knockouts.
\end{abstract}

\section{INTRODUCTION}

Following the sequencing of the mouse genome, it is now possible to create transgenic small animal models for the study of origin, progression, and therapy of human diseases. A number of large scale programmes are currently underway to create knockout models for each of the approximately 25000 mouse genes. Conventional screening methods for the detection and classification of morphological changes, such as histological microscopy, are timeconsuming and often limited at establishing the true penetration of a specific genotype into anatomical or functional abnormalities. As a result, there is enormous interest in the life sciences in developing new imaging tools that can characterise these models with high spatial resolution at a cellular and molecular level.

Photoacoustic imaging has shown great promise as a small animal imaging technique since it combines the high spatial resolution of ultrasound imaging with the high spectral specificity of optical techniques. The preferential absorption of visible and near-infrared light by haemoglobin allows the visualisation of vasculature to centimetre depths. This has been used to obtain images of the vascular structure in the brain [1], subcutaneous tumours and skin [2] in mice and rats. In addition, the spectroscopic specificity of tissue chromophores can be exploited to make spatially resolved measurements of their concentration through the use of multiple excitation wavelengths. This allows not only the monitoring of haemodynamic parameters, such as blood oxygen saturation [3], but also the detection of exogenous contrast agents. Using models of photoacoustic signal generation, it has also been shown that chromophore concentrations can be measured quantitatively [4]. Apart from exogenous contrast agents, the absorption provided by genetically expressed fluorescent proteins can also be visualised using photoacoustic spectroscopy [5]. By measuring the relative strength of these chromophore accumulations, information about specific cellular processes can be obtained.

While photoacoustic imaging has promising clinical applications, such as breast imaging, much recent research has been directed towards preclinical studies in small animals. The application of interest in this study is the structural and functional phenotyping of transgenic mice for the study of the genetic origins of vascular malformations, such as heart defects. For this application, magnetic resonance imaging (MRI) is one of the most widely used imaging modalities as it provides images of the anatomical structure of soft tissue. Although MRI is capable of obtaining hemodynamic information and a relative measure of oxygen saturation in tissue, it is challenging to obtain the high temporal and spatial resolution required for imaging the mouse embryo. In addition, while optical contrast agents are readily available for investigations of molecular processes in the embryo, MR agents with equivalent sensitivity are still in development. Photoacoustic imaging is therefore highly complementary to MRI as tissue contrast is largely due to the

Photons Plus Ultrasound: Imaging and Sensing 2010: The Eleventh Conference on Biomedical Thermoacoustics, Optoacoustics, and Acousto-optics, edited by Alexander A. Oraevsky, Lihong V. Wang, Proc. SPIE, Vol. 7564, 75641A (2010); doi:10.1117/12.842204 
strong optical absorption by blood and has the potential to measure blood oxygenation and to detect exogenous contrast agents in the mouse embryo in vivo. In this study, photoacoustic images of mouse embryos have been acquired to study the development of the vasculature following specific genetic knockouts.

\section{METHODS}

An optical parametric oscillator (OPO) laser system (Newport Spectra-Physics/GWU) provided 7 ns excitation pulses at $590 \mathrm{~nm}, 630 \mathrm{~nm}, 680 \mathrm{~nm}$ at a pulse repetition rate of $50 \mathrm{~Hz}$. The output of the OPO laser was coupled into a fused silica optical fibre (1.5 $\mathrm{mm}$ diameter) to homogenise the beam. The output of the fibre illuminated the embryo, which was placed on top of the photoacoustic scanner as shown in Figure 1 and immersed in a 2\%vol Intralipid (Fresenius Kabi) suspension to induce optical scattering and therefore a reasonably homogeneous incident light distribution. A forward mode detection geometry (Figure 1a) was used for whole body imaging, while backward mode detection (Figure 1b) was used to acquire high resolution images of the superficial vasculature. The beam diameter at the surface of the suspension was approximately $1.5 \mathrm{~cm}$ and resulted in fluences less $10 \mathrm{~mJ} \mathrm{~cm}{ }^{-2}$ which are lower than the maximum permitted exposure for skin. The acoustic waves were detected using a photoacoustic scanning system [6]. Its ultrasound sensing mechanism is based on the interferometric detection of acoustically induced changes in the optical thickness of a Fabry-Perot polymer film interferometer. The sensor was read out by scanning a focussed cw interrogation beam across its surface. The maximum scan area was $18 \mathrm{~mm}$ x $12 \mathrm{~mm}$, the largest step size was $100 \mu \mathrm{m}$. At each position of the scan, a photoacoustic signal was recorded without signal averaging. From these signals, 3D images of the initial pressure distribution were obtained using a Fourier transform based reconstruction algorithm [7].

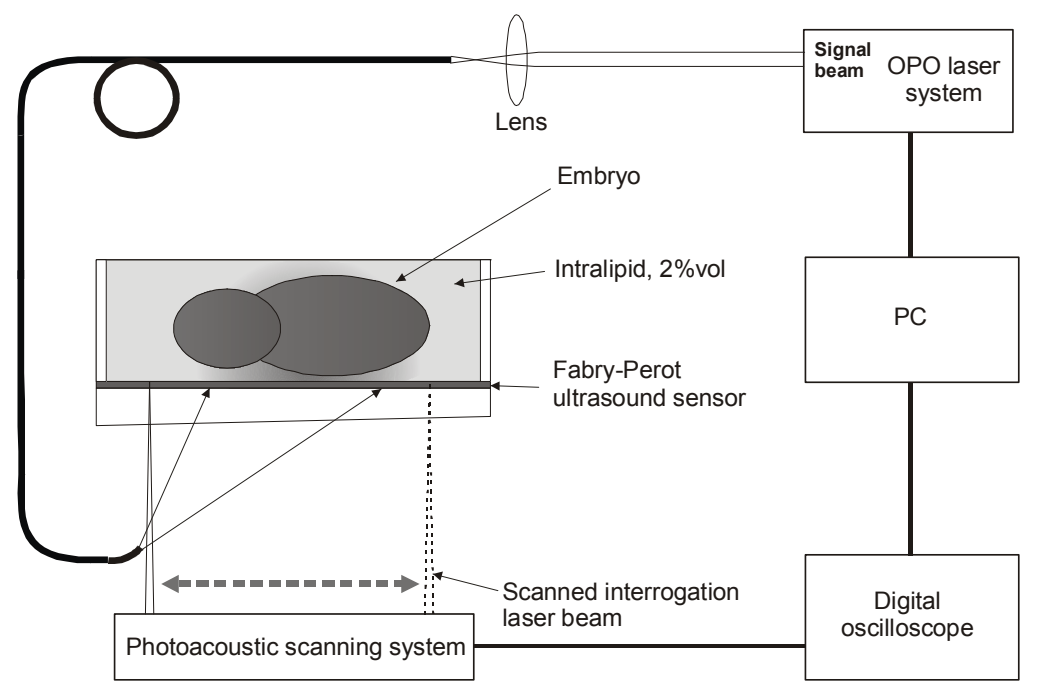

Figure 1 The experimental setup for the acquisition of 3D photoacoustic images of mouse embryos using backward mode detection.

Pregnant female mice ( $\mathrm{n}=1 \mathrm{CD}-1$ or $\mathrm{n}=1 \mathrm{Chd} 7+/$ - genetrap mutant (C57Bl6 background)) were sacrificed when their embryos reached 18.5 days. Chd7 is a gene implicated in the human condition CHARGE syndrome, which is characterised in part by heart defects. In this study, however, the embryos were imaged to demonstrate the capabilities of photoacoustic technology rather than to compare wild-type and mutant animals. Embryos were dissected and placed in Hanks solution (HBSS, Sigma-Aldrich) and their umbilical cords tied with a suture to preserve the circulating blood supply. The embryos were imaged within less than $1 \mathrm{~h}$ of sacrifice. The duration of each scan was approximately 5 minutes. 


\section{RESULTS}

Figure 2 shows the horizontal intensity projections of a 3D photoacoustic image of the upper body of an embryo. The vasculature of the head (such as the superior sagittal sinus), neck, upper body, and front legs, is clearly visible (Figure 2). The photoacoustic images obtained using forward mode detection are characterised by high signal strength across the entire thickness of the embryo. This was due to a fortuitous cancellation of the losses associated with acoustic attenuation on one hand and the depth dependent optical attenuation on the other.

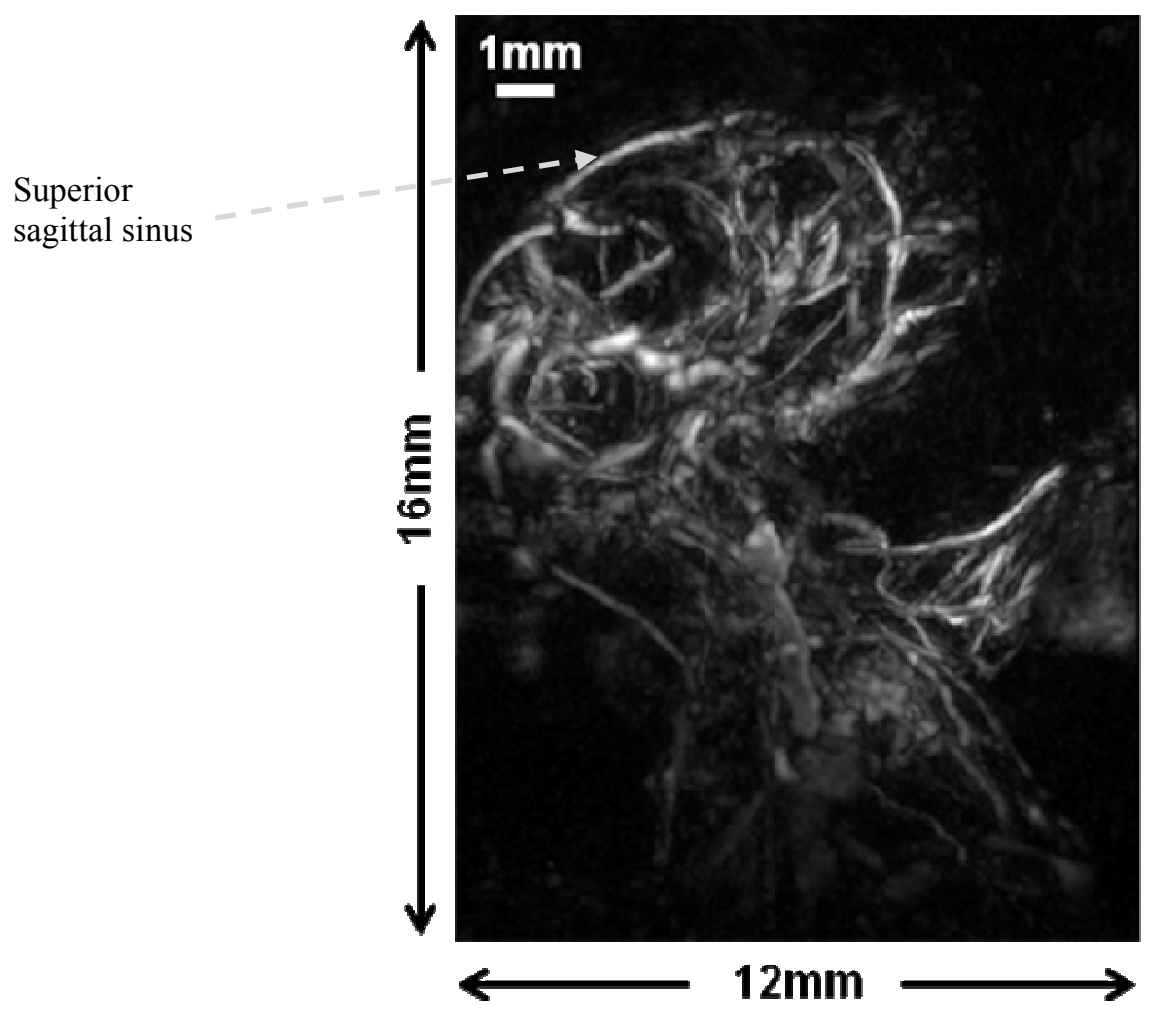

Figure 2 Maximum intensity projections of a 3D photoacoustic image of the head and upper body region of an ex vivo mouse embryo in vertical view.

This allowed the acquisition of images of the entire body including regions, such as the area around the heart, that are of high interest in the study of the genetic origins of vascular malformation. The same procedure was used to image the whole embryo, as shown in Figure 3, which appears to visualise blood perfused regions around the developing spine. Using a backward mode detection geometry (Figure 1) and by scanning over a smaller area $(7 \mathrm{~mm} \times 7 \mathrm{~mm})$ with smaller step sizes $(50 \mu \mathrm{m})$, high resolution images of the superficial vasculature were also obtained (Figure 4$)$. 

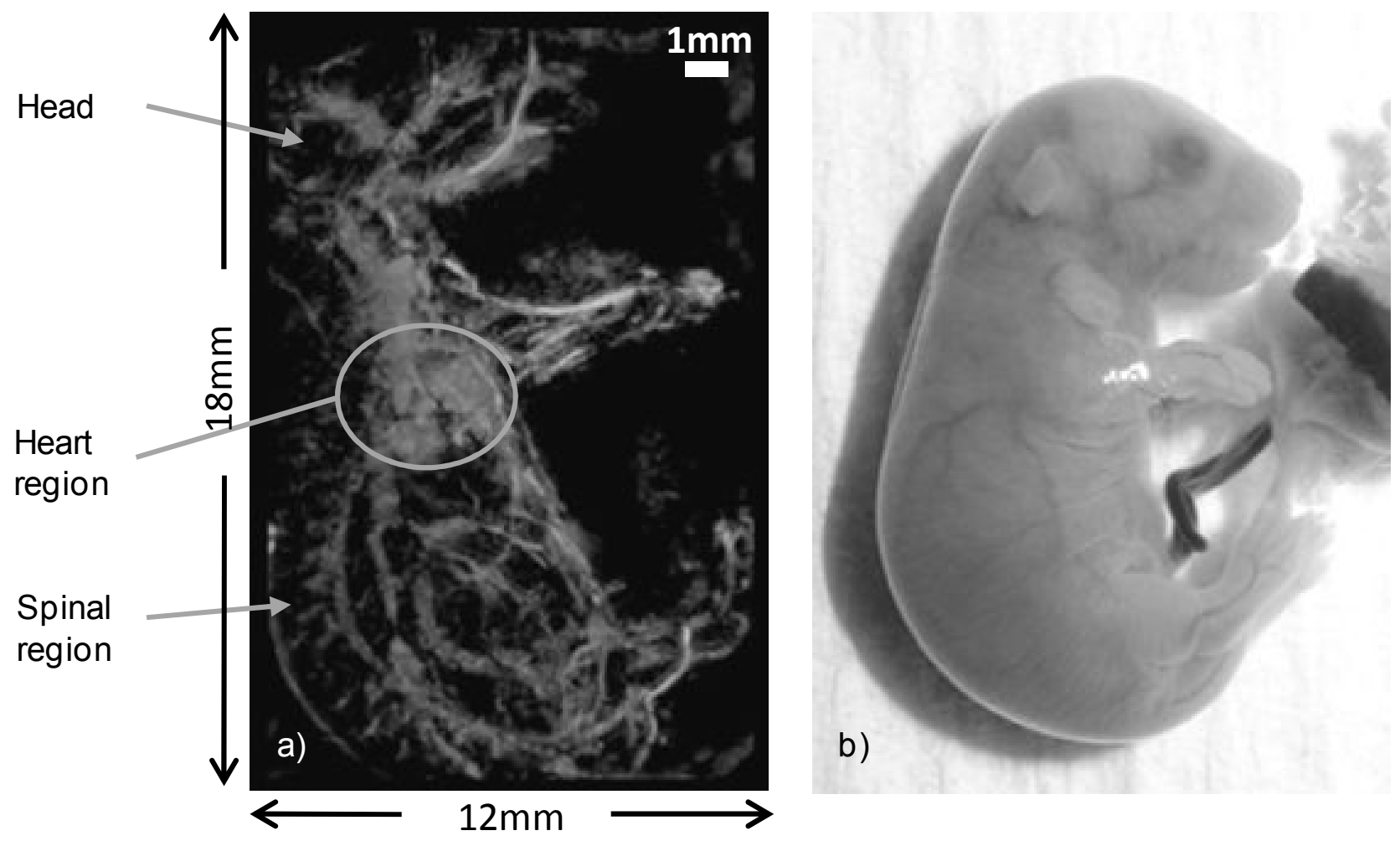

Figure 3 (a) Vertical maximum intensity projection of a 3D photoacoustic image and (b) a photograph of an ex vivo mouse embryo.

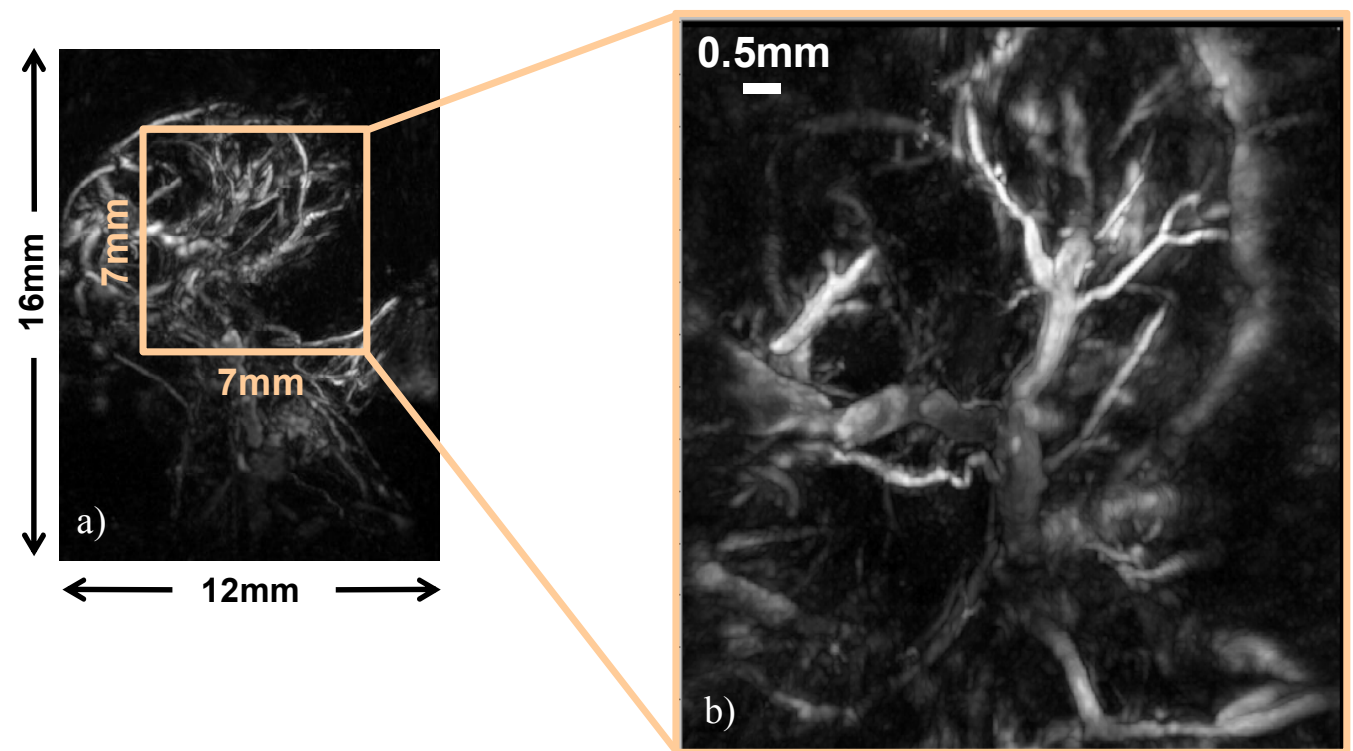

Figure 4 By scanning the head region of the embryo over a smaller area (Figure 4a) with smaller step sizes, high resolution images of the superficial vasculature were obtained - shown here as maximum intensity projection (b). 


\section{DISCUSSION}

Photoacoustic imaging has been shown to provide high resolution, whole body images of the vascular structure of transgenic mouse embryos. This may enable studies of the effects of genetic knockouts on the development of vascular malformations to be made. Given that mouse embryos are located less than $10 \mathrm{~mm}$ beneath the skin of the mother animal, it should be possible to acquire in vivo images of the embryo in situ. By acquiring images at multiple excitation wavelengths and by using model-based inversion methods, it may then be possible to monitor functional parameters, such as blood oxygenation, and to detect exogenous contrast agents in embryos in vivo.

\section{REFERENCES}

1. Laufer, J., et al., Three-dimensional noninvasive imaging of the vasculature in the mouse brain using a high resolution photoacoustic scanner. Applied Optics, 2009. 48(10): p. D299-D306.

2. Zhang, E.Z., et al., In vivo high-resolution 3D photoacoustic imaging of superficial vascular anatomy. Physics in Medicine and Biology, 2009. 54: p. 1035-1046.

3. Stein, E.W., K. Maslov, and L.H.V. Wang, Noninvasive, in vivo imaging of blood-oxygenation dynamics within the mouse brain using photoacoustic microscopy. Journal of Biomedical Optics, 2009. 14(2): 202502.

4. Laufer, J.G., et al., Quantitative determination of chromophore concentrations from photoacoustic images using a model-based inversion technique. Applied Optics, in press, 2010.

5. Razansky, D., et al., Multispectral opto-acoustic tomography of deep-seated fluorescent proteins in vivo. Nature Photonics, 2009. 3(7): p. 412-417.

6. Zhang, E., J. Laufer, and P. Beard, Backward-mode multiwavelength photoacoustic scanner using a planar Fabry-Perot polymer film ultrasound sensor for high-resolution three-dimensional imaging of biological tissues. Applied Optics, 2008. 47(4): p. 561-577.

7. Köstli, K.P. and P.C. Beard, Two-Dimensional Photoacoustic Imaging by Use of Fourier-Transform Image Reconstruction and a Detector with an Anisotropic Response. Applied Optics, 2003. 42(10): p. 1899-1908. 